[261] P. WeINStein. Z. Untersuch. Lebensm., 1934, 68, 73.

[262] R. Weissmann. Contr. et. réfractométrie lait de vache. Th. Doct. Sc., Naney, 1933.

[263] Wetham et Hammond. Journal Dairy Science, 1935, 6, 341-351.

[264] H. R. Whitehead et G. A. Cox. Biochem. Journal, 1933, 27, 951.

[265] Whitnah et Caulfield. Rep. Kans. Agric, Exp. Stat., 1932-34, pp. $83-5$.

[266] R. Wiliot. Milehw. Forsch., 1925, 2, 249-259.

[267] J. WiLly. Journal Ministry Agric., 1923, 29, 894.

[268] Winter et Parmentier. Rev. Gén. Lait, 1903-04, 3, 9-193.

[269] Wood. Journal Biol. Chem., 1905.

[270] M. E. Woodmann. Bioch. Journal, 1921, 15, 187.

[271] M. E. Woodmann et J. Hammond. Journal Agr. Science, 1922, 12.

[272] J. ZАYKowskY. Biochem. Z., 1924, 146, 189.

[273] Chr. ŹBInden. Le Lait, 1931, 11, 113.

\title{
L'ANALYSE CHIMIQUE DES COLLES EN POUDRE A BASE DE CASÉINE
}

\author{
TEAN PIEN \\ par \\ Directeur \\ G. RENVOISE \\ Chimiste
}

Laboratoires de la Laiterie des Fermiers Réunis

Les colles en poudre et autres produits similaires que l'on peut. préparer en partant de la caséine lactique offrent une très grande variété de composition et de propriétés.

D) nombreuses substances chimiques étant susceptibles d'entrer dans ces formules et leurs proportions respectives pouvant être mod fiées suivant le but à a'teindre, il en résulte une infinité de combinaisons possibles.

L'analyse chimique de ces mólanges présente donc un intérêt certain, mais auszi de réelles difficultés. C'est pourquoi nous avons cru devoir procéder à l'étude systématique de cette question.

$$
\text { *** }
$$

\section{ELÉMENTS A DOSER. EXAMEN QUALITATIF PRÉALABLE}

Les colles en poudre à base de caséine lactique sont des mélanges où l'on rencontre trois ou quatre (rarement davantage) des substances suivantes : caséine lactique, gélatine en poudre, chaux éteinte, carbonate de calcium, carbonate de sodium, fluorure de sodium, borate de sodium, phosphates de sodium, silicate de sodium silicate d'aluminium (rarement), silice libre (sous forme de quartz pulvérulent), sulfate de baryum (comme charge). 
Si l'on décompose les sels minéraux de cette liste en groupes d'ions, on voit qu'on peut avoir à doser (en dehors des protides eux-mêmes) :

Cations : Calcium, sodium, baryum, aluminium.

Anions : acides carbonique, fluorhydrique, borique, phosphorique, silicique, sulfurique.

On sait que le dosage de plusieurs de ces éléments ne peut pas être mené de la même façon suivant que l'on se trouve en présence ou en l'absence de certains autres. La présence simultanée des fluorures, borates, silicates pose, à cet égard, des problèmes particuliers.

Il est done absolument nécessaire, avant de commencer l'analyse quantitative d'une colle quelconque à base de caséine, d'en déterminer avec certitude la composition qualitative complète, ou du moins de s'assurer de la présence ou de l'absence de.chacun dès éléments énumérés ci-dessus.

\section{Séparation des substances organiques et des substances minérales}

La colle à examiner comporte toujours une partie organique, constituée le plus souvent par de la caséine lactique seule, et une partie minérale. Bien que la plupart des dosages des éléments minéraux doivent s'effectuer sur les cendres de la colle totale, nous recommandons vivement de commencer l'examen de la colle par une séparation - que l'on peut rendre quantitative pour des fins de vérification - des éléments organiques et des éléments minéraux. Cette opération est d'ailleurs indispensable pour le dosage de la gélatine s'il s'en trouve.

Le principe de la séparation repose sur l'emploi d'un liquide ne réagissant avec aucun des composants de la colle et d'une densité intermédiaire entre celle des deux groupes de substances à séparer. Après un certain nombre d'essais nous avons choisi le chloroforme.

\section{MODE OPERATOIRE}

10 grammes de colle en poudre sont introduits dans une ampoule à décantation avec environ $100 \mathrm{~cm}^{3}$ de chloroforme technique. On agite soigneusement. On place l'ampoule dans la position verticale et on débarrasse la partie supérieure de la paroi interne de l'ampoule des particules qui $y$ adhèrent en faisant couler sur cette paroi une petite quantité de $\mathrm{CHCl}^{3}$. Puis on abandonne au repos.

Très rapidement la caséine (et autres matières organiques éven- 
tuellement présentes) gagnent la surface. Les matières minérales se rassemblent toutes au fond. Quand le liquide qui sépare ces deux couches est devenu parfaitement limpide, on recueille la partie minérale sur filtre taré, on sèche et pèse. On obtient ainsi, avec une approximation suffisante, le taux de matières minérales de la colle.

Les résultats obtenus par cette méthode sont toujours légèrement trop faibles sur des colles un peu anciennes. Nous avons remarqué, en effet, que les colles, même à l'état de poudre sèche, subissent une certaine maturation spontanée. A la faveur de l'humidité toujours apportée par la caséine ou par l'atmosphère, il se produit un commencement de réaction entre certaines matières minérales (chaux surtout) et la caséine. On le demontrerait aisément en dosant les cendres de la caséine séparée par le chloroforme : elles augmentent aveo le temps tandis que le taux de matières minérales séparables diminue. Exemple : sur un mélange renfermant $70 \%$ de caséine et $30 \%$ de matières minérales, l'extraction chloroformique a permis de retrouver :

$29,92 \%$ de matières minérales pour une colle préparée le jour même. $29,80 \%$ de matières minérales pour une colle de cinq jours.

$28,97 \%$ de matières minérales pour une eolle de quinze jours.

Cette technique permet done de se faire une idée suffisamment exacte de la teneur globale de la colle en matières minérales. Les deux groupes de substances ainsi séparés sont utilisés pour l'analyse qualitative.

\section{Examen qualitatif de la partie organique}

Les matières organiques qui se trouvent rassemblées à la partie supérieure du chloroforme sont recueillies et examinées. En général elles sont constituées exclusivement par de la caséine lactique. Cependant certains fabricants utilisent dans la préparation des colles des mélanges de caséine et de gélatine (certaines colles en poudre ne renferment que de la gélatine). Il est done nécessaire d'identifier ces deux constituants seuls ou mélangés.

Lès réactions chimiques basées sur la mise en évidence du tryptophane de la caséine pourraient, àıla rigueur, servir à distinguer l'un de l'autre ces deux protides employés sepls. Il va de soi qu'elles sont inutilisables dans le cas de mélanges caséine + gélatine.

Mais il existe un moyen qualitatif très simple qui permet de trancher la question : la gélatine est aisément soluble dans l'eau tiède, alors qúe la caséine y est insoluble. 


\section{MODE OPERATOIRE}

On prélève environ 2 grammes des matières organiques séparées par le chloroforme et séchées. On les mélange dans un bécher avec 50 à $100 \mathrm{~cm}^{3}$ d'eau tiède. On agite pendant quelques instants.

En présence de gélatine seule, la solution est totale et le liquide prend le plus souvent une coloration jaune; il possède en outre une nette adhésivité dont on se rend parfaitement compte en évaporant une goutte de solution entre les doigts.

En présence de caséine seule, les grains de matière organique restent insolubles et tombent au fond du vase. Le liquide surnageant, quoique parfois légèrement trouble en raison de l'entraînement possible d'une petite quantité de matières minérales, reste incolore et ne possède aucune adhésivité.

En présence du mélange caséine + gélatine les caractères précédents se superposent : grains insolubles de caséine aru fond du vase, coloration jaune en général et nette adhésivité du liquide surnageant (dues à la gélatine).

Cette technique grossière ne permettrait évidemment pas de discerner la présence d'une petite quantité de gélatine dans un gros excès de caséine. Mais ce cas ne se présente jamais dans les colles de caséine où la gélatine, quand elle existe, figure toujours pour un pourcentage élevé.

Pratiquement, cet essai nous donne satisfaction et nous permet à peu près à coup sûr, de conclure à la présence éventuelle de. gélatine.

Dans le cas où cet essai qualitatif paraîtrait insuffisant, il serait, bien entendu, facile de le remplacer par le dosage de ces deux formes de matières azotées (voir plus loin).

\section{Remarque}

Il est très rare que les colles en poudre à base de caséine renferment de l'amidon ou de la dextrine. Un simple essai à l'eau iodée permettrait en tout cas de s'assurer de la présénce ou de I'absence de ces matières hydrocarbonées.

\section{Examen qualitatif de la partie minérale}

\section{A. IDENTIFICATION DES ANIONS}

\section{$1^{0}$ Silice des silicates solubles.}

La matière minérale séparée par le chloroforme et séchée est reprise par une solution chaude de carbonate de soude à $10 \%$. On amorce ainsi la précipitation de la chaux sous forme de carbonate sans que le silicate de chaux, moins insoluble, puisse se former. On ajoute alors de l'acide chlorhydrique, goutte à goutte en agitant 
jusqu'à neutralité exacte. La précipitation de la chaux 'est alors à peu près complète_et les silicates solubles restent en solution (1.)

On filtre. Le résidu sur filtre contient, entre autres, la silice libre insoluble et les silicates insolubles préexistants. Le filtrat contient, entre autres, les silicates solubles et certains sels métalliques.

La silice des silicates solubles ne peut pas être séparée par un traitement acide suivi de dessiccation comme dans la plupart des méthodes classiques. En présence de fluorure, il se formerait de l'acide hydrofluosilicique volatil qui exporterait la silice que l'on se propose précisément de rechercher. On procède done différemment :

Le filtrat est traité à chand par le carbonate d'ammoniaque (on ajoute au filtrat bouillant une solution saturée de carbonate d'ammoniaque. On maintient environ une heure à l'ébullition en remplaçant le carbonate d'ammoniaque vaporisé). Au cours de cette opération, la silice des silicates solubles précipite en flocons. On sépare le précipité par filtration. On lave (les fluorures solubles passent dans. le filtrat).

Le résidu insoluble est repris par del'acide chlorhydrique au 1/2, On évapore ensuite à sec dans les conditions habituelles et on porte à l'étuve pour insolubiliser la silice. On reprend une dernière fois par de l'acide chlorhydrique dilué. On ajoute alors une assez grande quantité d'eau. Seule, la silice constitue l'insoluble.

S'il ne subsiste, après cette reprise par l'eau, aucun insoluble, on peut affirmer que la colle ne contenait pas de silicates solubles.

\section{$2^{\circ}$ Silice des silicates insolubles et silice libre (quartz,} terre d'infusoires, ....).

Dans l'essai précédent le premier précipité formé (carbonate de chaux) séparé par filtration, contient toutes les formes de silice insoluble éventuellement présentes.

Leur identification se fera de la manière suivante : ce précipité est lavé à fond, séché, calciné. On le soumet ensuite à la fusion alcaline dans les conditions ordinaires avec du carbonate double de potassium et de sodium.

Au cours de cette fusion la silice libre et les silicates insolubles forment du silicate de soude soluble : la première par combinaison directe, les seconds par double décomposition. Après fusion, on reprend par l'eau le contenu du creuset de platine. Il se sépare un précipité (formé principalement de carbonate de chaux) ; la solution renferme du silicate de soude provenant de toutes les formes de

(1) L'usage de l'oxalate d'ammoniaque n'est pas reeommandable pour les raisons exposées plus loin. 
silice insoluble et, le cas échéant, de l'alumine, sous forme d'aluminate de soude, provenant des silicates d'alumine éventuellement présents dans la colle. On filtre.

L'étude du filtrat nous ramène au cas précédent (recherche des silicates solubles) : on traite par le carbonate d'ammonium à l'ébullition. La silice précipite. Le résidu, séparé, est repris par $\mathrm{HCl}$ au 1/2. On évapore à sec pour insolubiliser la silice, etc. Si la liqueur finale de reprise par l'eau du résidu desséché fait apparaître un insoluble, on peut affirmer qu'on se trouve en présence de silice provenant, cette fois, des silicates insolubles ou de la silice libre contenus dans la colle.

\section{$3^{\circ}$ Fluor (des fluores alcalins ou alcalins-terreux).}

De nombreuses colles de caséine renferment du fluorure de sodium.

On connaît la méthode elassique qui permet d'identifier le fluor dans un mélange minéral : dans une capsule de platine on mélange le produit à examiner réduit en poudre avec un peu d'acide sulfurique concentré de manière à constituer une bouillie claire. On recouvre d'une plaque de verre enduite de vernis de graveur peu fusible ; à l'aide d'une pointe fine on aura tracé des traits dans la couche de vernis. On chauffe doucement. Sous l'influence de l'acide sulfurique, l'acide fluorhydrique des fluorures est libéré et attaque le verre à l'emplacement des traits ménagés dans le vernis. Au bout d'une heure, on retire la plaque, fond le vernis et nettoie à l'essence. Si les traits sont devenus visibles, le produit à examiner contenaìt des fluorures.

Les résultats fournis par l'application de cette technique ne sont utilisables que s'ils sont positifs. L'absence de réaction positive

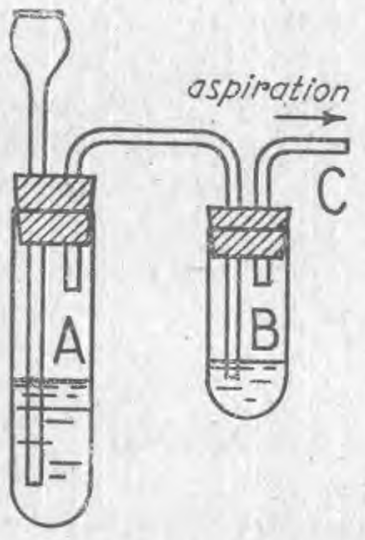
ne signifie pas nécessairement absence de fluorures ; en effet : en présence de silice ou de silicates, l'acide fluorhydrique qui a tendance à se former donne du fluorure de silicium $\mathrm{SiF}^{4}$ qui n'attaque pas le verre.

Si les essais qualitatifs précédents ont fourni la preuve de la présence de silice ou de silicates (solubles ou non) la technique classique de recherche du fluor he convient done plus et il faut avoir recours à la technique suivante plus générale :

Un gramme environ de la matière à examiner (matières minérales extraites de la colle à l'aide du chloroforme) est mélangé à un gramme environ de sable quart- 
zeux ou de verre pilé dans le tube A de l'appareil représenté ciavant. Le tube B contient 2 à $3 \mathrm{~cm}^{3}$ d'eau. Par l'entonnoir du tube $\mathrm{A}$ on introduit quelques centimètres cubes de $\mathrm{SO}^{4} \mathrm{H}^{2}$ à $66^{\circ}$ Bé.

On aspire légèrement par le tube $\mathrm{C}$ et on chauffe doucement le tube A.

En présence d'un fluorure, et grâce à l'adjonction de silice, il se -forme $\mathrm{SiF}^{4}$ qui se décompose au contact de l'eau du tube $\mathrm{B}$ et abandonne de la silice gélatineuse insoluble. En l'absence de fluorures cette réaction est évidemment négative.

\section{Remarques}

a) Il faut que le tube A, l'entonnoir et le tube de liaison soient parfaitement secs pour éviter une décomposition prématurée du $\mathrm{SiF}^{4}$.

b) Il y a intérêt à ce que l'appareil soit aussi petit que possible (les tubes $\mathrm{A}$ et $\mathrm{B}$ seront, constitués par des segments de tubes à essai de petit diamètre).

\section{$4^{\circ}$ Bore.}

L'essai classique à la flamme verte donne ici de bons résultats. Voici comment il convient de procéder :

Dans une petite capsule de porcelaine on introduit quelques grammes de matières minérales extraites de la colle. On y ajoute quelques centimètres cubes d'alcool méthylique puis un peu d'acide sulfurique. On mélange avec un agitateur de verre et on enflamme dans un endroit sombre. En présence de bore la flamme est nettement frangée de vert et cette coloration est assez persistante.

\section{$5^{\circ}$ Acide phosphorique.}

La matière minérale extraite est additionnée d'un excès d'acide nitrique au $1 / 3$. On laisse digérer quelques minutes au bain-marie en agitant. On dilue et on filtre. Au filtrat on ajoute, dans les conditions ordinaires, un excès de réactif nitromolybdique. L'apparition d'un précipité jaune (facilitée éventuellement pár un chauffage de quelques minutes à $60-70^{\circ}$ ) dénote la présence de phosphates (solubles ou insolubles).

\section{$6^{\circ}$ Acide carbonique (carbonates).}

L'addition d'acide dilué à la matière minérale fournit une effervescence qui suffit ici à affirmer la présence de carbonates. En cas de doute, faire barbotter le gaz dégagé dans un peu d'eau de baryte. 


\section{B. IDENTIFICATION DES CATIONS}

\section{$1^{\circ}$ Calcium.}

La matière minérale séparée par le chloroforme est reprise par $\mathrm{HCl}$ au 1/3. On dilue; on filtre. Le filtrat est rendu ammoniacal. S'il se forme un précipité (phosphates, hydrates métalliques) on le dissout par un apport d'acide acétique et on ajoute (après une nouvelle fil ${ }^{+}$ration s'il y a lieu) une petite quantité d'oxalate d'ammoniaque saturé. En présence de chaux il se forme un précipité dont l'apparition est facilitée par chauffage.

\section{$2^{\circ}$ Baryum.}

Dans les colles de caséine le baryum n'existe guère que sous la forme de sulfate de baryte, introduit à titre de charge inerte.

La recherche de ce sel doit toujours être effectuée et on y procédera comme suit :

Les matières minérales de la colle, traitées par un acide dilué pour la recherche du calcium, ont laissé un insoluble' (en l'absence de cet insoluble, il n'y a pas à rechercher le sulfate de baryte). L'insoluble séparé par filtration, soigneusement lavé et séché, est soumis à la fusion alcaline en creuset de platine. Au cours de cette fusion le sulfate de -baryte donne du carbonate de baryte insoluble et du sulfate de soude soluble. Après refroidissement on reprend par l'eau, filtre l'insoluble qui est lavé avec soin à l'eau chaude.

Dans le filtrat on peut identifier les sulfates par addition de $\mathrm{BaCl}^{2}$.

Le résidu insoluble, bien lavé, est traité sur le filtre par $\mathrm{HCl}$ au $1 / 10^{e}$ qui le dissout, au moins en partie. Dans la solution ainsi obtenue le baryum passé à l'état de chlorure est identifié par un léger apport de $\mathrm{SO}^{4} \mathrm{H}^{2}$ dilué.

Toutes ces opérations peuvent être effectuées quantitativement - ce qui mène directement au dosage du baryum. Le dosage des sulfates (s'il n'y a pas d'autres ions $\mathrm{SO}^{4}$ que ceux de sulfate de baryte) apportera la confirmation de celui du baryum.

\section{$3^{\circ}$ Sodium.}

Toutes les colles de caséine contiennent des sels de sodium. Il est donc inutile d'en effectuer la recherche qualitative qui serait toujours positive. Mais, dans tous les cas, il conviendra de doser ce métal par la méthode exposée plus loin.

\section{$4^{\circ}$ Autres métaux.}

Il est assez rare qu'on ait à se préoccuper de la présence d'autres métaux que les alcalins et alcalino-terreux dans les colles.

La présence éventuelle d'aluminium (charges de kaolin, etc.) 
aura été signalée lors de la recherche des silicates. Celle du cuivre (utilisé quelquefois comme agent imperméabilisant des colles) sera rendue évidente par la coloration bleue des solutions, notamment après la reprise par l'eau des fusions alcalines.

$$
\text { ** * }
$$

\section{ANALYSE QUANTITATIVE}

Indépendamment du dosage des éléments dont l'examen qualitatif vient de nous révéler la présence, il convient d'effectuer à part le dosage des matières minérales globales par calcination.

Lors de la séparation des substances minérales par le chloroforme, nous avons vu que l'on pouvait procéder à un véritable dosage de ces substances dans leur état initial.

La détermination des cendres apporte des éléments d'information nouveaux. La calcination fait perdre l'eau de constitution de certains sels, décompose les carbonates alcalino-terreux en oxydes. On dispose de la sorte de moyens supplémentaires pour parvenir à la reconstitution exacte du produit examiné.

\section{Dosage des matières minérales par calcination}

On calcine 5 grammes de colle en poudre en ayant soin de chauffer doucement au début pour éviter la fusion prématurée de certains sels de soude - ce qui aurait pour résultat d'enrober des particules charbonneuses impossibles à brûler par la suite.

Ce dosage est assez inexact ; car si l'on chauffe assez fort pour décomposer les carbonates alcalino-terreux, on risque de perdre certains sels alcalins relativement volatils; si l'on chauffe assez peu pour conserver ces derniers, on n'obtient pas la décomposition des carbonates alcalino-terreux - que, cependant, on pourra avoir amorcée suivant la température atteinte.

Quoiqu'il en soit, le taux de "cendres" est toujours inférieur de $10 \%$ ou davantage à celui des matières minérales réelles séparées au chloroforme (perte des molécules d'eau de cristallisation, décomposition partielle des carbonates, etc.)

Une méthode qui, sans être parfaite, donne cependant des résultats plus constants et plus faciles à interpréter, consiste à faire les cendres sulfuriques en utilisant le mode opératoire suivant :

On chauffe doucement, au rouge très sombre, 5 grammes de colle en poudre. Quand la masse est devenue charbonneuse on laisse refroidir, on humecte d'acide nitrique et on porte au bain de sable. Quand la masse est presque à sec, on ajoute une petite quantité d'acide sulfurique et on continue le chauffage au B. S. jusqu'à disparition complète des fumées blanches. Enfin on porte au moufle, au rouge sombre, jusqu'à cendres blanches. 
$\mathrm{Au}$ cours de cette opération, les sels alcalins dont on pouvait redouter la perte partielle à la calcination sont passés à l'état de sulfate de sodium très stable vis-à-vis de la chaleur.

C'est donc là une méthode de choix pour la préparation du dosage du sodium. En outre, ce chiffre de cendres sulfuriques sera précieux pour contrôler par le caleul la reconstitution de la colle en fin d'analyse.

\section{Dosage des matières organiques azotées}

Le problème consiste essentiellement à doser la caséine et la gélatine si l'examen qualitatif a fait soupçonner la présence de cette dernière. On procédera de la manière suivante :

10 Dosage de l'azote total;

$2^{\circ}$ Dosage de la caséine débarrassée de la gélatine;

$3^{\circ}$ La gélatine s'obtient par différence.

(Il n'existe jamais, dans les colles de caséine, de protides solubles dans l'eau autres que la gélatine.)

\section{$1^{\circ}$ Dosage de l'azote total.}

On utilise la méthode Kjeldahl classique en employant le cuivre comme catalyseur. On aurait sans doute avantage à faire usage de catalyseurs plus actifs tels que le sélénite de mercure dont l'emploi tend à se répandre. Mais, pour le but poursuivi ici, la précision donnée par le cuivre est largement suffisante et l'extrême facilité du dosage dans ces conditions nous a incités, jusqu'à plus ample informé, à conserver notre technique initiale qui est la suivante :

2 grammes de colle en poudre sont introduits dans un matras Kjeldahl avec 10 grammes de sulfate de potasse et 1 gr. 5 de sulfate de cuivre cristallisé. On ajoute $25 \mathrm{cc}$. d'acide sulfurique pur à $66^{\circ}$ Bé. On chauffe doucement en évitant l'ascension des mousses. Quand la matière a cessé de mousser, on augmente le chauffage et on maintient en douce ébullition jusqu'à décoloration de la masse. On poursuit cette ébullition encore une demi-heure après avoir obtenu un liquide parfaitement limpide. On distille ensuite dans les conditions habituelles en présence de $100 \mathrm{~cm}^{3}$ de lessive de soude (sans sulfure de sodium, puisque le catalyseur employé ici ne forme pas de complexes azotés). On recueille le distillat dans $20 \mathrm{~cm}^{3}$ d'acide sulfurique $\mathrm{N} / \mathrm{l}$ en présence de rouge de méthyle. On titre en retour par $\mathrm{NaOH} N / 1$. $1 \mathrm{~cm}^{3} \mathrm{SO}^{4} \mathrm{H}^{2} \mathrm{~N} / 1$ saturé au cours de la distillation correspond à 0 gr. 014 d'azote. Le taux de matières protéiques s'obtient en multipliant le taux d'azote trouvé par le coefficient 6,39 . 


\section{$2^{\circ}$ Dosage de la caséine seule.}

On part des matières organiques séparées par le chloroforme. Après avoir chassé les matières minérales rassemblées à la partie inférieure de l'ampoule à décantation, on reçoit les matières organiques surnageantes sur un filtre plat. On rince l'ampoule à plusieurs reprises avec du chloroforme. On sèche et pèse. Après quoi on rassemble la totalité de ces substances dans une capsule. On les mélange intimement. On en prélève 2 grammes que l'on introduit dans un bécher avec $100 \mathrm{~cm}^{3}$ d'eau chaude légèrement chlorhydrique $(0,2 \%$ environ) afin de dissoudre les traces de chaux éventuellement entraînées. On laisse en contact pendant quelques minutes en agitant avec soin. La gélatine se dissout. On sépare par filtration la caséine restée insoluble. On lave à fond ce résidu de caséine jusqu'à ce que les eaux de lavage ne contiennent plus de matières organiques. Le filtre et son contenu sont ensuite introduits dans un matras Kjeldahl et on procède à une attaque et au dosage de l'azote comme dans le cas de l'azote total.

Le taux de matières protéiques obtenu ici correspond à la caséine seule. On en déduit le taux de caséine lactique industrielle en multipliant le résultat par le coefficient 100/83. La plupart des caséines lactiques titrent en effet de 82 à $84 \%$ de matières protéiques.

\section{$3^{\circ}$ Taux de gélatine.}

Du chiffre de matières protéiques totales on retranche le taux de matières protéiques correspondant de la caséine. On obtient ainsi le taux de matières protéiques correspondant à la gélatine. On en déduit le taux de gélatine industrielle en multipliant le résultat par le coefficient 100/95. Los gélatines industrielles utilisées dans la préparation des colles titrent, en effet, environ $95 \%$ de matières protéiques.

\section{EXEMPLE D'APPLICATION PRATIQUE}

Nous avons prépáré un mélange à parties égales de caséine lactique en poudre et de gélatine en poudre dont les teneurs en azote étaient les suivantes :

Azote \% Matières protéiques \%

$\begin{array}{lll}\text { Caséine lactique } \ldots \ldots \ldots \ldots \ldots & 12,97 & 82,87 \\ \text { Gélatine } \ldots \ldots \ldots \ldots \ldots \ldots \ldots & 14,84 & 94,82\end{array}$

L'applieation des techniques précédentes a fourni les résultats suivants : 
a) Azote total du mélange.

$\begin{array}{lrc} & \text { Azote } \% & \text { Matières protéiques } \% \\ \text { Chiffre théorique } \ldots \ldots \ldots \ldots \ldots & 13,90 & 88 ; 82 \\ \text { Chiffre trouvé } \ldots \ldots \ldots \ldots \ldots & 13,80 & 88,18\end{array}$

b) Dosage de la caséine seule (après élimination de la gélatine).

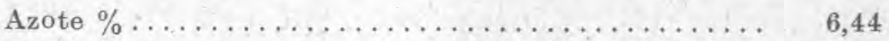

Matières protéiques correspondantes .......... 41,15

Caséine lactique correspondante........... 49,6

(Contre $50 \%$, chiffre théorique.)

c) Calcul du taux de gélatine.

Azote $\%: 13,80-6,44 \cdot=\ldots \ldots \ldots \ldots \ldots \ldots \ldots \ldots .7,7,36$

Matières protéiques correspondantes .......... 47,03

Gélatine correspondante ................ 49,

(Contre $50 \%$, chiffre théorique.)

Cette technique de séparation fournit donc des résultats très satisfaisants et peut être considérée comme correcte pour le but poursuivi ici.

\section{Dosage des anions}

\section{$1^{0}$ Dosage de la silice totale.}

La présence éventuelle de silicates difficilement attaquables et surtout la présence possible (et très fréquente) de fluorures, interdit l'emploi des méthodes d'attaque acide (il se formerait du fluorure de silicium volatil).

Il faut donc, dans tous les cas, faire appel à la méthode classique de la fusion alcaline qui permet de transformer les silicates insolubles et la silice libre en silicates solubles.

On commence par faire les cendres ordinaires de 5 grammes de colle en poudre. On réduit ces cendres en poudre très fine et on les soumet à la fusion alcaline en creuset de platine avec 12 grammes de $\mathrm{CO}^{3} \mathrm{KNa}$. On maintient au rouge vif pendant une demi-heure au moins, en remuant de temps en temps avec le fil de platine. La masse refroidie est reprise par l'eau bouillante. On se trouve alors en présence d'un précipité et d'une solution. On leș sépare par filtration sur filtre sans cendres et on lave à fond à l'eau chaude le résidu insoluble. Ce résidu (que nous appellerons $R_{1}$ ) contient surtout du carbonate de chaux et des traces de silice.

Le filtrat et les eaux de lavage contiennent (avec la totalité du fluor sous forme de NaF soluble) la silice à l'état de silicate de soude soluble. On le sature à froid par $\mathrm{NO}^{3} \mathrm{H}$ jusqu'à réaction légèrement acide et on abandonne plusieurs heures. $\mathrm{CO}^{2}$ se dégage. On ajoute ensuite une solution saturée de carbonate d'ammoniaque dans 
le but de précipiter la silice. On porte et maintient à l'ébullition pendant au moins une heure en remplaçant de temps en temps le $\mathrm{CO}^{3} \mathrm{Am}^{2}$ vaporisé $(1)$.

La silice qui précipite dans ces conditions est obtenue en flocons plus fins et plus denses que ceux que l'on obtient dans la méthode classique (en ne procédant pas à la neutralisation nitrique préalable). La séparation et le lavage de ce précipité de silice sont ainsi rendus plus faciles. En outre nous avons vérifié que cette manière de faire entraînait une précipitation pratiquement complète de la silice ce qui rend inutile l'emploi ultérieur de l'hydroxyde de zinc recommandé par M. BonIs (2). Après refroidissement on sépare sur filtre sans cendres le précipité ainsi obtenu (résidu $R_{2}$ ). Le filtrat est conservé pour le dosage du fluor.

Les deux résidus $R_{1}$ et $R_{2}$ contiennent entre autres choses la totalité de la silice. On les sèche complètement. On les introduit avec leurs filtres dans une capsule de porcelaine. (On aura pris soin de faire tomber dans la capsule le contenu du filtre $R_{2}$ et de le pulvériser avec un agitateur à tête plate; sinon, après caleination, la silice devenue très dure résisterait à la pulvérisation. On ajoute ensuite dans la capsule le filtre $R_{2}$ puis le filtre $R_{1}$ et son contenu.) On calcine doucement, d'abord au rouge sombre en évitant l'inflammation du filtre. Puis on chauffe plus fort et, quand la masse est devenue blanche, on laisse refroidir.

On écrase alors la matière et on reprend par de l'acide chlorhydrique au 1/3. On évapore à see au bain-marie en ayant soin de broyer la masse au fur et à mesure de la dessiccation. On maintient la capsule pendant douze heures à $100^{\circ}$. On reprend par $\mathrm{HCl}$ au $1 / 2$ et on évapore de nouveau au bain-marie en broyant finement la masse pendant la dessiccation. On maintient encore douze heures à $100^{\circ}$. La silice est ainsi complètement insolubilisée.

On ajoute alors $10 \mathrm{~cm}^{3}$ d'acide chlorhydrique au $1 / 2$ puis environ $50 \mathrm{~cm}^{3}$ d'eau chaude. On agite soigneusement la masse et on porte au bain-marie bouillant pendant quelques minutes pour bien dissoudre les sels. On jette ensuite sur filtre taré, on lave avec soin d'abord à l'eau bouillante légèrement chlorhydrique, puis avec de l'eau distillée chaude.

Si l'insolubilisation a été bien conduite, la filtration est rapide. Le filtrat et les eaux de lavage sont reeueillis. On y dosera l'aluminium éventuellement présent et la chaux.

(1) La technique qui consiste à reprendre par un acide le produit de la fusion alcaline et à aller à sec pour insolubiliser la silice ne peut pas non plus être appliquée ici en raison de la présence possible de fluorures.

(2) A. Bonis. Dosage de fluosilicate de baryum dans les poudres insectioides. Annales falsif., 1935, p. 461. 
Le résidu de silice sur filtre est séché à fond et calciné avec les précautions habituelles (chauffer doucement en évitant l'inflammation du filtre ; après carbonisation chauffer plus fort et, quand le résidu est devenu. blanc, chauffer au rouge vif pendant un quart d'heure.)

Après refroidissement au dessiccateur, on pèse et l'on en déduit le taux de $\mathrm{SiO}^{2}$ totale de la colle.

\section{Remarque}

Ce traitement acide, qui est à proserire pour l'attaque directe des cendres de la colle en raison de la présence possible de fluorures, peut être effectué sans inconvénients sur les résidus $R_{1}+R_{2}$ qui sont exempts de fluor.

\section{$2^{\circ}$ Dosage de la silice soluble (silicate de soude)}

On part des matières minérales séparées au chloroforme dont on connaît le taux global. Après avoir éliminé le chloroforme, on mélange bien ces substances et on en pèse 1 ou 2 grammes.

On introduit cette prise d'essai dans un bécher; on y ajoute un excès de solution chaude de carbonate de soude à $10 \%\left(20\right.$ à $30 \mathrm{~cm}^{3}$ pour 1 gramme de matières minérales) dans le but d'amorcer la précipitation de la chaux (1) sans que le silicate de chaux puisse se former. On neutralise par addition de HCl. On maintient au bain-marie un temps suffisant pour permettre la séparation complète du précipité de carbonate de chaux et la dissolution du silicate de soude ( 1 ou 2 heures.). On filtre et lave. Le résidu contient la silice libre et les silicates insolubles. Le filtrat renferme les silicates solubles (et éventuellement les fluorures solubles).

On traite ce filtrat par le carbonate d'ammoniaque dans le but de précipiter la silice, comme dans le cas précédent (dosage de la silice totale après fusion alcaline). A cet effet, on ajoute au filtrat une solution saturée de carbonate d'ammoniaque. On porte et maintient à l'ébullition pendant au moins une heure en remplaçant de temps en temps le $\mathrm{CO}^{3} \mathrm{Am}^{2}$ vaporisé. On sépare par filtration le précipité de silice qui s'est formé. On lave, sèche et calcine. Le résidu de silice obtenu est repris par. $\mathrm{HCl}$ au $1 / 3$ et on achève le dosage par insolubilisation en milieu acide comme indiqué plus haut. Le poids obtenu correspond à la silice des silicates solubles.

\section{EXEMPLES D'APPLICATION PRATIQUE}

Sur des colles à base de caséine préparées par nous et renfer-

(1) L'usage de l'oxalate d'ammoniaque n'est pas à recommander. Il s'en suit ultérieurement une gêne dans la préeipitation de la silice par le earbonate d'ammoniaque à l'ébullition. 
mant : silicate de soude, chaux, fluorures, borates, carbonates, nous avons obtenu les résultats șuivants pour le silicate de soude :

$\begin{array}{lcccc} & 1 & 2 & 3 & 4 \\ \text { Chiffre théorique } \ldots . & 20,00 & 20,00 & 16,66 & 16,66 \\ \text { Trouvé à l'analyse } \ldots & 19,56 & 19,62 & 16,25 & 16,32\end{array}$

\section{Remarque}

Certains silicates de soude en poudre sont difficilement ou incomplètement solubles dans l'eau. La méthode précédente conduit done parfois à des résultats déficitaires pour la silice soluble. Aussi quand la colle ne contient pas à la fois silicates solubles et silicates insolubles - (ce qui est le cas général) convient-il mieux de traiter la matière minérale comme dans le cas du dosage de la silice totale (fusion alcaline) même s'il n'y a que des silicates solubles. On n'utilisera la méthode précédente que si l'on se trouve à la fois en présence de silicates solubles et insolubles.

$3^{\circ}$ Dosage de la silice insoluble (silice libre ou silice des silicates insolubles).

Le taux de silice insoluble s'obtient par différence.

On peut aussi, dans le but d'obtenir une vérification des dosages précédents, la doser directement en reprenant le précipité formé ci-dessus par traitement au carbonate de soude des matières minérales séparées au chloroforme (dosage de la silice soluble). Ce résidu contient en effet du carbonate de chaux, la silice libre et les silicates insolubles. On le lave, sèche et calcine. On le soumet à la fusion alcaline et on achève le dosage comme dans le cas du dosage. de la silice totale.

\section{$4^{\circ}$ Dosage du fluor.}

Après avoir essayé diverses méthodes (notamment la méthode Frésénius modifiée par A. CARNot que nous ne sommes pas parvenus à rendre d'un emploi commode et sûr dans le cas des colles de caséine) nous avons finalement adopté, en la modifiant légère. ment, la méthode préconisée par M. Bonis pour l'analyse des fiuosilicates insolubles (I).

La première partie de cette technique consiste à séparer la silice. On opère comme il vient d'être dit (dosage de la silice totale) : on fait les cendres ordinaires de 5 grammes de colle. On les soumet à la fusion alcaline. On reprend par l'eau, filtre et lave, neutralise le filtrat jusqu'à légère acidité par $N O^{3} H$ et précipite la silice par le carbonate d'ammoniaque à l'ébullition. Après séparation du résidu

(1) A. BonIs, (Loe. cit.). 
$\mathrm{R}_{2}$ le filtrat contient la totalité du fluor sous forme de fluorure de sodium soluble.

Ce filtrat alcalin est ramené à une légère acidité par $\mathrm{HCl}$ dilué en présence d'hélianthine, puis à une légère alcalinité par addition de 1 ou $2 \mathrm{~cm}^{3}$ de carbonate de soude à $10 \%$. On porte ce liquide au voisinage de l'ébullition et on y verse lentement une solution de chlorure de calcium qui précipite le fluor sous forme de $\mathrm{CaF}^{2}$ (ainsi que les earbonates et phosphates éventuellement présents sous forme de sels de Ca correspondants). Après 10 minutes d'ébullition, on laisse reposer. On décante. On recueille le précipité sur filtre sans-cendres, (on conserve le filtrat pour le dosage du bore éventuellement présent). Le filtre et le précipité qu'il contient, lavés à l'eau chaude, sont ensuite traités dans un bécher par de l'acide acétique à $10 \%$ qui dissout les carbonates et phosphates de calcium sans attaquer le $\mathrm{CaF}^{2}$. Après digestion de quelques minutes au bainmarie, le résidu insoluble exclusivement constitué par le papier du filtre et par $\mathrm{CaF}^{2}$ est jeté sur un nouveau filtre. lavé, séché, calciné et pesé.

\section{EXEMPLES D'APPLICATION PRATIQUE}

Sur des colles de caséino préparées spécialement et contenant du fluor sous forme de fluorure de sodium ou de fluorure de calcium, nous avons obtenu les résultats suivants :

$\begin{array}{lcccc} & 1 & 2 & 3 & 4 \\ \text { Chiffre théorique } \ldots . . & 15,00 & 33,30 & 16,60 & 27,50 \\ \text { Trouvé à l'analyse } \ldots & 14,45 & 32,21 & 16,15 & 26,30\end{array}$

Ls résultats obtenus (qui ne sont pas parfaits en raison des nombreuses causes d'erreur que présente le dosage du fluor en présence de silice, bore, chaux, phosphates, etc.) sont néanmoins suffisants pour le but poursuivi ici.

\section{$5^{\circ}$ Dosage du bore.}

On part des cendres ordinaires de 5 grammes de colle en poudre. On procède à l'élimination de la silice comme dans le cas du dosage de la silice totale : fusion alcaline, reprise par l'eau, neutralisation par $\mathrm{NO}^{3} \mathrm{H}$, précipitation de la silice par $\mathrm{CO}^{3} \mathrm{Am}^{2}$.

Le filtrat du résidu $\mathrm{R}^{2}$ contient tout le fluor et tout le bore (éventuellement présents) à l'état soluble.

Doux eas peuvent se présenter :

a) L'essai qualitatif n'a pas révélé la présence de fluorures : le dosage du bore est alors effectué directement sur lefiltrat du résidu $\mathbf{R}_{2}$ (dosage de la silice totale).

b) L'essai qualitatif a révélé la présence de fluorures : on procède 
alors au dosage de ces derniers comme il a été indiqué ci-dessus et on effectue le dosage du bore sur le filtrat du précipité formé par addition de chlorure de calcium et contenant le $\mathrm{CaF}^{2}$ (voir plus haut).

Il convient tout d'abord de se débarrasser de la chaux contenue dans ces filtrats. Celui qui correspond au cas (a) n'en contient que des traces, car la majorité de la chaux a été retenue dans le précipité $\mathrm{R}_{1}$. Celui qui eorrespond au cas (b) en contient des quantités importantes puisqu'on a introduit $\mathrm{du} \mathrm{CaCl}^{2}$ en excès pour former $\mathrm{CaF}^{2}$.

Dans les deux cas on porte la liqueur à l'ébullition et on y ajoute de l'axalate d'ammoniaque en excès. On l'abandonne quelques heures au bain-marie. On filtre et lave à l'eau chaude.

On se trouve donc en présence d'une solution débarrassée de la silice, du fluor et de la chaux qu'elle pouvait renfermer. On y dosele bore parla technique suivanteissue de la méthode de Stromeyer (1) dont le principe réside dans la formation de fluoborate de potassium insoluble dans une solution concentrée d'acétate de $\mathbf{K}$.

On transvase la sólution contenant le bore dans une capsule de platine.' Il est inutile, contrair $€$ ment à ce que recommande StROMEYer, d'ajouter de la potasse. Le $\mathrm{CO}^{3} \mathrm{KNa}$ qui a servi à la fusion alcaline des cendres a apporté une quantité de potassium suffisante à la formation de $\mathrm{BF}^{3}$. KF.

On ajoute alors un excès de $\mathrm{HF}$ bien exempt de $\mathrm{SiO}^{2}$. On évapore à sec au bain-marie. Il faut qu'il y ait assez d'acide fluorhydrique pour que, au cours de l'évaporation, on constate nettement le dégagement de vapeurs acides. Le résidu sec contient le fluoborate de $K$ et du fluorure acide de $K$ en excès (KF.HF).

On reprend, à froid, ce résidu par une solution agueuse d'acétate de potassium à $20 \%$ dans laquelle le fluoborate de $K$ est insoluble, alors que le fluorure acide de $\mathrm{K}$ se dissout. On laisse digérer quelques heures en agitant de temps en temps. On filtre sur filtre taré. On lave avec une solution à $20 \%$ d'acétate de $\mathrm{K}$ pour éliminer KF.HF jusqu'à ce que le filtrat ne fournisse plus de précipité par addition de $\mathrm{CaCl}^{2}$. On achève le lavage avec de l'alcool à $84 \%$, on sèche et pèse.

100 parties de $\mathrm{BF}^{3} \mathrm{KF}$ correspondent à 27,76 parties de $\mathrm{B}^{2} \mathrm{O}^{3}$. En multipliant le poids du précipité de $\mathrm{BF}^{3} \mathrm{KF}$ par 0,758 on obtient le poids de borax correspondant (biborate de sodium hydraté : $\mathrm{B}^{4} \mathrm{O} \mathrm{\textrm {Na } ^ { 2 }}, 10 \mathrm{H}^{2} \mathrm{O}$ ).

\section{EXEMPLES D'APPLICATION PRATIQUE}

Sur les colles de caséine préparées spécialement et contenant

(1) Citée dans Chesneav, p. 284 (Principes théoriques et pratiques d'analyse minérale. Béranger, éditeur). 
du borax (en présence ou en l'absence de fluorures) nous avons obtenu :

$\begin{array}{lcccc} & & 1 & 2 & 3 \\ \text { Chiffre théorique } \ldots \ldots \ldots \ldots & 16,66 & 16,66 & 50,00 \\ \text { Trouvé à l'analyse } \ldots \ldots \ldots & 15,87 & 17,01 & 48,23\end{array}$

$6^{\circ}$ Dosage des carbonates.

Nous avons essayé les diverses méthodes classiques. Cell e qui nous a fourni les meilleurs résultats consiste à noter la perte de

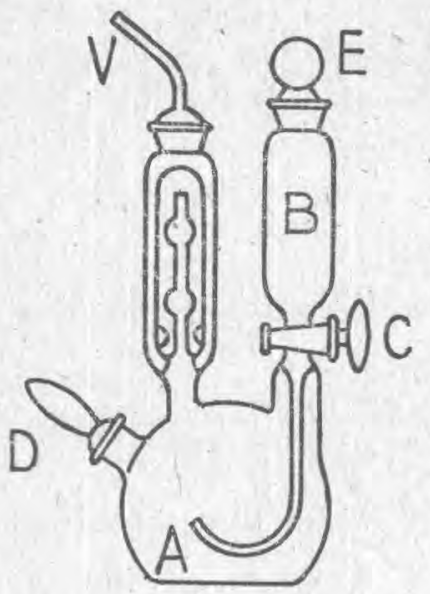
poids du mélange après traitement a l'acide ehlorhydrique en utilisant, de préférence, le petit appareil de ScHRcedTER ou celui de ROHRBECK.

On prendra soin d'employer de l'acide au $1 / 3$. Un acide plus concentré peut entraîner un dégagement de chaleur capable de provoquer des pertes de vapeur d'eau.

On place 1 gramme de colle en poudre en $\mathrm{A}$. On ferme $\mathrm{C}$ et on garnit B aux $3 / 4$ d'acide chlorhydrique au $1 / 3$. On ferme $D$ et $E$ et on pèse le tout (P gr.).

On débouche $\mathrm{E}$ et on tourne $\mathrm{C}$ de manière que l'acide s'écoule lentement en A. On agite doucement pour bien humecter toute la matière. Lorsque tout l'acide s'est écoulé, on ferme $\mathrm{C}$ et $\mathrm{E}$. On agite encore l'appareil. Quand toute effervescence a cessé, on fait un léger vide en aspirant en $\mathrm{V}$ pendant quelques secondes de manière à libérer $\mathrm{CO}^{2}$ qui reste dissous dans le liquide chlorhydrique ; puis on ouvre $\mathbf{E}$ et $\mathrm{C}$ en continuant d'aspirer légèrement pendant 10-15 secondes pour purger l'appareil. On ferme alors $\mathrm{E}$ et $\mathrm{C}$ et on pèse ( $\mathrm{P}^{\prime}$ gr.).

P-P' donne le poids de $\mathrm{CO}^{2}$ contenu dans un gramme de colle.

\section{EXEMPLES D'APPLICATION PRATIQUE}

Dans un produit renfermant $0 \mathrm{gr}$. 5 de $\mathrm{Co}^{3} \mathrm{Ca}$ on a retrouvé, en appliquant cette technique, 0 gr. 498.

\section{$7^{\circ}$ Dosage des phosphates.}

Le dosage des phosphates dans les colles de caséine ne présente aucune difficulté particulière. On opérera soit sur les matières minérales séparées au chloroforme (dont on prélèvera une partie aliquote) soit sur la colle entière elle-même, mais non sur les cendres 
en raison de la perte possible (dans certains cas) d'une partie du phosphore à l'état de phosphures pendant la calcination.

On effectuera une attaque sulfonitrique, suivie d'une précipitation à la mixture magnésienne dans les conditions classiques que nous rappellerons brièvement :

2 grammes de matière sont introduits dans un petit ballon d'attaque avec $10 \mathrm{~cm}^{3}$ d'acide nitrique et $20 \mathrm{~cm}^{3}$ d'acide sulfurique. Attaquer à l'ébullition jusqu'à décoloration en rajoutant s'il y a lieu, à plusieurs reprises, de l'acide nitrique. Quand l'attaque est terminée (ce qui ne demande que 10 à 20 minutes en l'absence de caséine) laisser refroidir, diluer à $200 \mathrm{~cm}^{3}$, filtrer, prélever $100 \mathrm{~cm}^{3}$ et ajouter $40 \mathrm{~cm}^{3}$ de citrate d'ammoniaque, $10 \mathrm{~cm}^{3}$ de mixture magnésienne, 70 à $75 \mathrm{~cm}^{3}$ d'ammoniaque et terminer le dosage dans les conditions ordinaires (agitation, repos de 12 heures, nouvelle agitation, filtration, lavage à l'eau ammoniacale, séchage, calcination et pesée à l’état de pyrophosphate de magnésie).

\section{Dosage des cations}

\section{Dosage du calcium.}

Il est indispensable, avant de procédèr à ce dosage, de faire disparaître la silice et les fluorures éventuellement présents.

Si les essais qualitatifs ont apporté la preuve de la présence de silice ou de silicates, on sera amené à doser la silice totale (donc à l'éliminer) et le dosage de la chaux s'effectuera sur le dernier filtrat de reprise acide de la silice insolubilisée (voir plus haut). Cette technique vaut également pour le cas de la présence simultanée de silice et de fluorures car ces derniers sont décomposés par le traitement acide qui termine le dosage de la silice.

Si les essais qualitatifs ont montré qu'on se trouve en présence de fluorures sans silice ou silicate, le problème est plus simple : on traite les cendres de 5 grammes de colle par 20 ou $25 \mathrm{~cm}^{3}$ d'acide ehlorhydrique au 1/3. On évapore à sec au bain-marie. On pulvérise le résidu et on le reprend de nouveau par 10 ou $20 \mathrm{~cm}^{3}$ d'acide chlorhydrique dilué en allant de nouveau à see. Dans ces conditions les fluorures sont décomposés et tous les sels de chaux sont dissous à l'état de chlorures. On reprend par l'eau chaude, on filtre, lave et on dose la chaux dans le filtrat.

Enfin si les essais qualitatifs ont montré qu'il n'y avait dans la colle ni silicates, ni fluorures on se conténtera de traiter les cendres de $5 \mathrm{gr}$. de colle par $\mathrm{HCl}$ au $1 / 3$ en agitant soigneusement et en chauffant quelques minutes au bain-marie pour bien dissoudre tous les sels de chaux. On filtre et lave à l'eau chaude. On dose la chaux dans le filtrat.

Dans tous les cas donc, on se trouve finalement en présence 
d'une solution acide, exempte de silice et de fluorures, dans laquelle toute la chaux (et éventuellement l'alumine) est dissoute à l'état de chlorure. On achève le dosage, comme suit :

Le filtrat est alcalinisé par l'ammoniaque puis légèrement réacidifié par l'acide acétique. En principe il redevient limpide ; s'il y a lieu, on filtre. On porte à l'ébullition et on ajoute quelques centimètres cubes d'une solution saturée chaude d'oxalate d'ammoniaque. On laisse reposer au bain-marie. Au bout de quelques heures on filtre sur filtre sans cendres, lave à l'eau chaude renfermant de l'oxalate d'ammoniaque, sèche et calcine. Quand le produit obtenu est blanc on porte au Meker pendant une demi-heure. On refroidit à l'exsiccateur et on pèse la chaux $(\mathrm{CaO})$ obtenue.

\section{EXEMPLES D'APPLICATION PRATIQUE}

Sur des colles de caséine renfermant de la chaux, des silicates, du fluorure de sodium, nous avons obtenu les résultats suivants :

$\begin{array}{llcccc} & & 1 & 2 & 3 & 4 \\ \text { Chiffre théorique de } \mathrm{CaO} & \ldots & 60,4 & 37,8 & 21,9 & 21,9 \\ \text { Trouvé à l'analyse } \ldots \ldots \ldots \ldots & 59,7 & 37,4 & 21,7 & 22,0\end{array}$

\section{$2^{\circ}$ Dosage du sodium.}

Nous avons adopté la méthode Ǩahane (1) qui donne d'excellents résultats. On part des cendres sulfuriques de 5 grammes de colle. On les reprend par de l'eau chaude en ayant soin de bien écraser la matière. On laisse digérer quelque temps au bain-marie et on décante sur filtre plat. Le filtrat est reçu dans une fiole jaugée de 200 (on lave à plusieurs reprises l'insoluble...).

\section{ELIMINATION DES PHOSPHATES}

La présence de quantités importantes d'ion phosphorique est une cause d'erreur dans le dosage du sodium par la méthode à l'acétate d'uranyle, car il forme, en présence de ce réactif, du phosphate d'uranyle insoluble. A vrai dire, de petites quantités de phosphore seraient sans inconvénient en présence de beaucoup de sodium, car à poids égal, l'ion sodium donne un précipité de complexe vingt-deux fois plus lourd que l'ion $\mathrm{PO}^{4}$. Néanmoins, si l'examen qualitatif a révélé la présence de phosphates en quantité non négligeable, il est recommandé de procéder à leur élimination de la manière suivante :

Dans la fiole de 200 contenant le filtrat et les eaux de lavage des cendres sulfuriques, ajouter de l'ammoniaque jusqu'à neutralité, puis quelques grammes de chlorure d'ammonium et quelques décigrammes de carbonate de magnésie (selon la méthode de Fiske,

(1) E. KанаNe. Bulletin de la Société Chimique de France, avril 1930, page 382. 
citée par KAHANE). Nous avons constaté qu'il y avait intérêt à attendre douze ou mieux vingt-quatre heures avant de filtrer. L'acide phosphorique est alors en grande partie insolubilisé à l'état de phosphate ammoniaco-magnésien. On complète alors à $200 \mathrm{~cm}^{3}$ et on filtre.

\section{PRÉCIPITATION DU SODIUM}

On prélève $10 \mathrm{~cm}^{3}$ du filtrat précédent (correspondant à 0 gr. 25 de colle). On les introduit dans un bécher de $250 \mathrm{~cm}^{3}$ avec $50 \mathrm{~cm}^{3}$ de réactif de Kahane. On agite pendant quelques minutes pour accélérer la précipitation et on laisse reposer une demi-heure. On décante sur creuset de verre fritté à pâte fine (correspondant par exemple au Schott No 4). On reprend le précipité par de l'alcool à $96^{\circ}$ saturé d'acétate triple d'uranyle, magnésium, sodium (provenant d'un dosage précédent). On entraîne tout le précipité sur le filtre et on lave avec cet alcool saturé puis avec 1 ou $2 \mathrm{~cm}^{3}$ d'alcool pur. On sèche à l'étuve (une demi-heure suffit) et pèsè. Le poids de précipité multiplié par 0,015 donne le poids de sodium (exprimé en $\mathrm{Na}$ ) de la prise d'essai.

(Si le poids de précipité dépassait 1 gramme il y aurait intérêt à recommencer le dosage en utilisant $5 \mathrm{~cm}^{3}$ de filtrat au lieu de 10.)

\section{PRÉPARATION DU RÉACtif dE KAHANE}

La composition du réactif est la suivante :

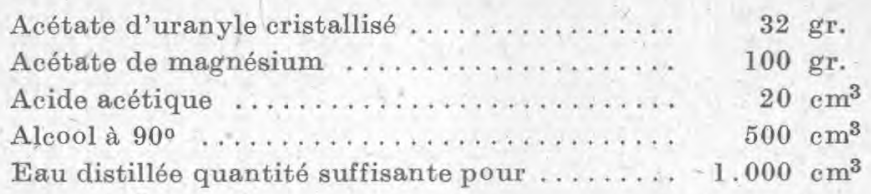

On prépare ce réactif en chauffant au bain-marie les acétates de magnésium et d'uranyle avec l'acide acétique, l'alcool et environ $300 \mathrm{~cm}^{3}$ d'eau. La dissolution s'effectue rapidement. On laisse refroidir, compète à $1.000 \mathrm{~cm}^{3}$ et abandonne au repos. Le réactif peut être utilisé après quelques heures de repos et après filtration. Il est cependant avantageux de le laisser reposer le plus longtemps possible afin d'éliminer les dernières traces de sodium.

\section{EXEMPLES D'APPLICATION PRATIQUE}

a) Sur des sels de sodium purs nous avons obtenu (en Na\%):

Théorique

$$
39,31
$$

Chlorure de sodium ..............

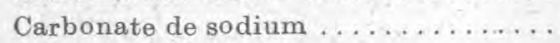

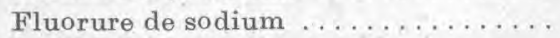

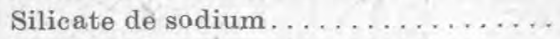

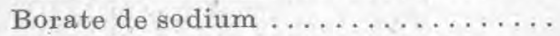

$$
43,39
$$
12,04
Trouvé à l'analyse 39,20 43,31 54,75 37,62 12,06 
b) Sur des colles de caséine renfermant des doses variées de sels de sodium divers nous avons obtenu par exemple :-

1

27,37

$\mathrm{Na} \%$ théorique ...........

Trouvé à l'analyse ........
2

24,64

24,32

Comme on le voit, la méthode K̇ahane appliquée aux colles de caséine conduit à des résultats remarquablement satisfaisants.

\section{$3^{\circ}$ Dosage du baryum.}

Le baryum, avons-nous dit, n'existe dans les colles de caséine qu'à l'état de sulfate de baryte au titre de charge.

On utilisera, pour ce dosage, le mode opératoire indiqué plus haut pour la recherche qualitative de l'ion Ba en s'attachant à rendre les opérations quantitatives.

Le dosage des sulfates auquel on procédera en même temps, conduira à une bonne vérification de celui du baryum. (Il n'y a jamais, dans les colles de caséine, d'autres ions $\mathrm{SO}^{4}$ que ceux du baryum, sauf dans le cas très rare où la colle contiendrait du sulfate de cuivre.)

\section{$4^{\circ}$ Dosage de l'aluminium.}

On effectue ce dosage sur la liqueur destinée au dosage de la chaux (voir plus haut) et débarrassée éventuellement de la silice et des fluorures. On y ajoutera, à chaud, quelques grammes de $\mathrm{NH}^{4} \mathrm{Cl}$ et un excès d'ammoniaque. On effectuera la précipitation et la séparation de l'alumine dans les conditions classiques. (C'est dans ce filtrat que l'on dosera la chaux.)

Il va de soi que si on ne désire pas connaître le taux d'alumine éventuellement présente, le dosage de la chaux peut être effectué sans séparation préalable de l'alumine en procédant comme il a été dit plus haut (addition successive d'ammoniaque, d'acide acétique et d'oxalate d'ammoniaque).

\section{Remarque}

En présence de phosphates le dosage de l'alumine est faussé car il précipite du phosphate d'alumine au lieu d' $\mathrm{Al}^{2} \mathrm{O}^{3}$. Il est rare que les colles de caséine contiennent des phosphates et encore plus rare qu'elles renferment des sels d'aluminium (silicates d'Al, kaolin, etc.). L'éventualité de la présence simultanée des phósphates et de sels d'aluminium dans les colles de caséine n'est done pas à envisager. Néanmoins, si ce cas se présentait, on précipiterait la totalité de l'alumine à froid à l'état de phosphate en ajoutant un excès de phosphate de soude à la solution ammoniacale-rendue 
acétique comme pour le dosage de la chaux. Il se forme un précipité blanc de phosphate d'alumine hydraté que l'on lave, sèche, calcine $\left(\mathrm{PO}^{4} \mathrm{Al}\right)$.

(Pour plus de détails concernant ce cas exceptionnel, voir Chesnéau : Principes d'Analyse Minérale, p. 369.)

\section{$*^{*} *$ \\ EXEMPLE COMPLET D'APPLICATION PRATIQUE}

Nous avons préparé une colle en poudre renfermant la totalité des substances susceptibles d'y être rencontrées c'est-à-direprésentant, pour l'analyse, le maximum de difficultés.

La composition de ce mélange était la suivante :

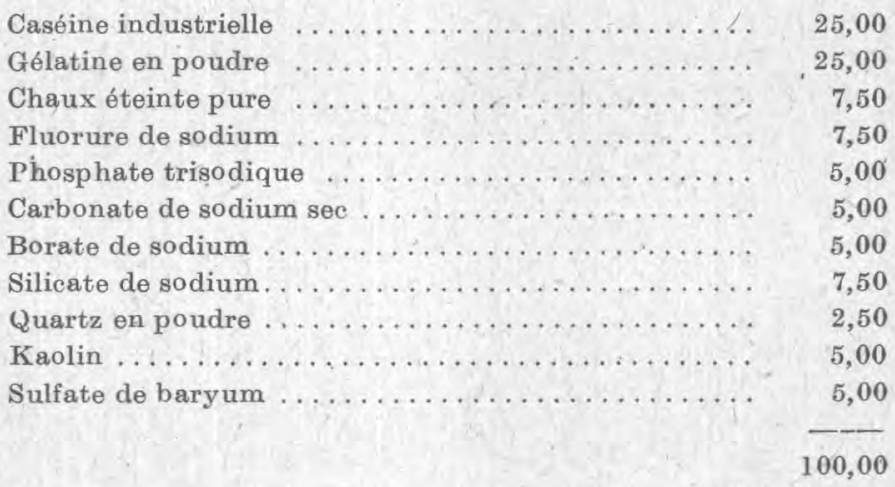

Nous avons effectué l'analyse complète de ce mélange en utilisant les méthodes exposées au cours de ce travail.

Voici les résultats que nous avons obtenus :

\section{Exprimés en éléments dosés \% du mélange total.}

\section{Chiffres réels}

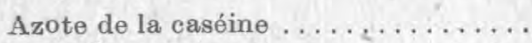

Azote de la gélatine ............

Silice totale .................

Silice soluble ................

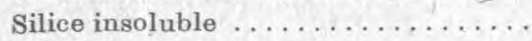

Fluor . . . . . . . . . . . .

Bore ....................

Carbonates $\left(e n \mathrm{CO}^{2}\right.$ ) ...........
(1)

3,30
3,80
8,56
3,63
4,93
3,36
0,60
1,98
0,99

Chiffres trouvés

à l'analyse du mélange

3,22
3,81
8,38
3,54
4,84
3,24
0,61
2,02
0,95

3,81

8,38

3,54

4,84

3,24

0,61

2,02

0,95

(1) D'après l'analyse effectuée de chacune des substances entrant dans la composition du mélange. 
Chiffres réels

(1)

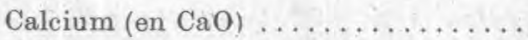

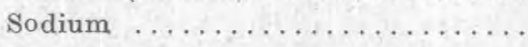

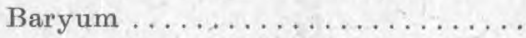

Aluminium (en $\mathrm{Al}^{2} \mathrm{O}^{3}$ )

$2^{\circ}$ Exprimés en substances reconstituées

D'après les

chiffres réels

Caséine industrielle $\ldots \ldots \ldots \ldots \ldots$.

Gélatine en poudre .............

Chaux éteinte pure .............

Fluorure de sodium .............

Phosphate trisodique $\left(\mathrm{a} 12 \mathrm{H}^{2} \mathrm{O}\right) \ldots$.

Carbonate de sodium anhydre ......

Borate de sodium (à $\left.10 \mathrm{H}^{2} \mathrm{O}\right) \ldots \ldots \ldots$

Silicate de sodium sec............

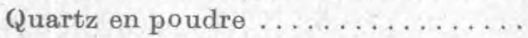

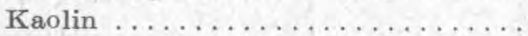

Sulfate de baryum .............
Chiffres trouvés à l'analyse du mélange

$$
\begin{array}{r}
5,46 \\
10,37 \\
2,75 \\
2,02
\end{array}
$$

par le calcul.

D'après les chiffres trouvés à l'analyse

\begin{tabular}{rr}
25,0 & 24,83 \\
25,0 & 25,62 \\
7,20 & 7,21 \\
7,43 & 7,15 \\
5,30 & 5,12 \\
4,77 & 4,87 \\
5,23 & 5,29 \\
7,39 & 7,17 \\
2,47 & 2,48 \\
5,14 & 5,18 \\
4,74 & 4,77 \\
\hline- & - \\
99,67 & 99,69
\end{tabular}

Comme on le voit, les méthodes d'analyse proposées donnent d'assez bons résultats, même dans le cas d'un mélange aussi complexe que celui-ci. Dans les cas courants (mélanges de 3 ou 4 substances au plus) l'analyse est beaucoup plus facile et les résultats obtenus sont encore meilleurs.

\title{
ANTAGONISIME DU COLIBACILLE ET DES BACTÉRIES PUTRIDES DANS LE LAIT CONTAMINÉ (2)
}

\author{
par
}

\section{A. ROCHAIX et F. SIMON}

On sait que parmi les microbes ayant une signification de contamination fécale, que l'on a l'habitude de rechercher dans les liquides comme l'eau, le lait, etc., se trouve au premier plan le colibacille, auquel on a adjoint depuis quelques années les "bactéries putrides". Ces dernières sont des microbes paraissant

(1) Voir note précédente, page 143.

(2) Ass. Microb. de langue française, 2-12-1943; Ann. Inst. Pasteur, 1944, LXX, 313. 